\title{
Novel tertiary amine oxide surfaces that resist non-specific protein adsorption
}

Suzanne J. Dilly, ${ }^{\text {a }}$ Matthew P. Beecham, ${ }^{\text {a }}$ Steven P. Brown, ${ }^{c}$ John M. Griffin, ${ }^{c}$

Andrew J. Clark, ${ }^{\text {a }}$ Craig D. Griffin, ${ }^{\mathrm{b}}$ Jacqueline Marshall, ${ }^{\mathrm{b}}$ Richard Napier, ${ }^{\mathrm{b}}$ Paul C.

Taylor, ${ }^{\mathrm{a}}$ Andrew Marsh. ${ }^{\mathrm{a} *}$

a Department of Chemistry, University of Warwick, Coventry, CV4 7AL, UK

b Warwick HRI, Wellesbourne, Warwick, CV35 9EF, UK

c Department of Physics, University of Warwick, Coventry, CV4 7AL, UK

\section{Supporting Information}

Tabulated data for Figure 4

\begin{tabular}{|c|c|c|c|c|c|c|c|c|c|c|}
\hline Support & PEGA & Tentagel & Lantern & Lantern & Lantern & Silica & Silica & Silica & Silica & Silica \\
\hline Cmpd & & & & $5 a$ & $8 a$ & & 9 & 10 & 13 & 14 \\
\hline \multirow{17}{*}{$\begin{array}{c}\mathrm{pfu} / \mathrm{ml} \\
20 \mathrm{mg} \\
\text { support }\end{array}$} & 225000 & 380 & 93000 & 800 & 6800 & 20300 & 164000 & 1640 & 660000 & 9400 \\
\hline & & 570 & 90000 & 900 & 7100 & 22000 & 54600 & 34700 & 220000 & 27400 \\
\hline & & 760 & 88000 & 1000 & 7300 & 23300 & 164000 & 21900 & 140000 & 11000 \\
\hline & & 750 & 104000 & 1300 & 7300 & 21100 & 54600 & 7940 & 148000 & 62700 \\
\hline & & 900 & 101000 & 1600 & 7300 & 23900 & 19820 & 9140 & & 79600 \\
\hline & & 750 & 96000 & 1700 & 1500 & 26800 & 21200 & & & \\
\hline & & 900 & 10000 & 47300 & 7300 & 102000 & & & & \\
\hline & & 3900 & 26000 & 55200 & 1200 & 62800 & & & & \\
\hline & & 10300 & 433000 & 37200 & 2400 & 82800 & & & & \\
\hline & & 10700 & 402000 & 8330 & 1700 & & & & & \\
\hline & & & 355000 & 11400 & 2200 & & & & & \\
\hline & & & 360000 & & 53200 & & & & & \\
\hline & & & 772000 & & 70200 & & & & & \\
\hline & & & 13600 & & 46400 & & & & & \\
\hline & & & 15300 & & 62400 & & & & & \\
\hline & & & & & 4150 & & & & & \\
\hline & & & & & 4140 & & & & & \\
\hline Mean & 225000 & 2994 & 197260 & 15158 & 17206 & 144788 & 79833 & 15057 & 292000 & 38019 \\
\hline S. d. & & 4093 & 219200 & 20864 & 23890 & 330200 & 67000 & 13220 & 248000 & 31620 \\
\hline
\end{tabular}


Note that standard deviation (s.d.) and hence error bar is not shown for PEGA resins because only one replicate was made and this material was not considered further.

\section{Additional solution phase syntheses.}

The preparation of 6-benzyloxy- $N, N^{\prime}$-bis(3-morpholin-4-ylpropylamine oxide) $[1,3,5]$ triazine is described. The straightforward addition of 4-(3aminopropyl)morpholine to 2-benzyloxy-4,6-dichloro[1,3,5]triazine was achieved at $60{ }^{\circ} \mathrm{C}$ in THF giving 6-benzyloxy- $N, N^{\prime}$-bis(3-morpholin-4-ylpropylamine oxide) (Scheme 1). The oxidation of the tertiary amines was then carried out using $m$-CPBA in dichloromethane at low temperature (Scheme 2). The solution was treated with 2methylpropene in order to remove any excess peracid. The solution ${ }^{13} \mathrm{C}$ signals of the final product were correlated with those for the supported amine oxides, providing evidence of the identity of the product on solid phase and completeness of the reaction.

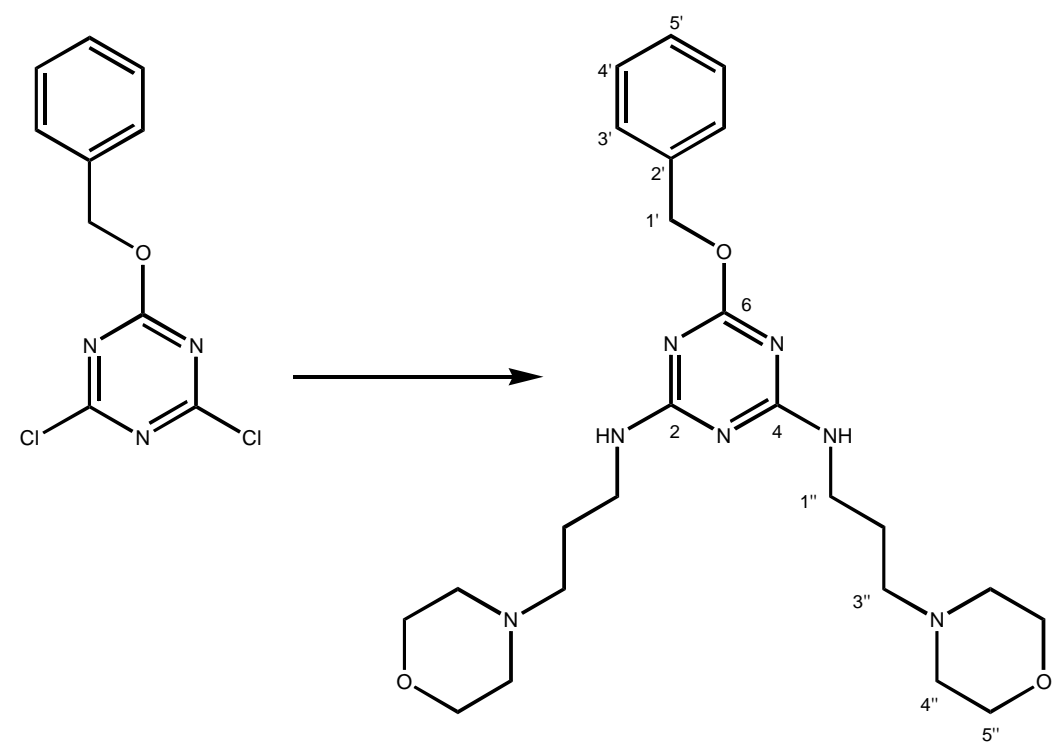

Scheme 1 Reagents and Conditions: 4-(3-aminopropyl)morpholine, THF, diisopropylethylamine, $60{ }^{\circ} \mathrm{C}, 16 \mathrm{~h}(59 \%)$. 


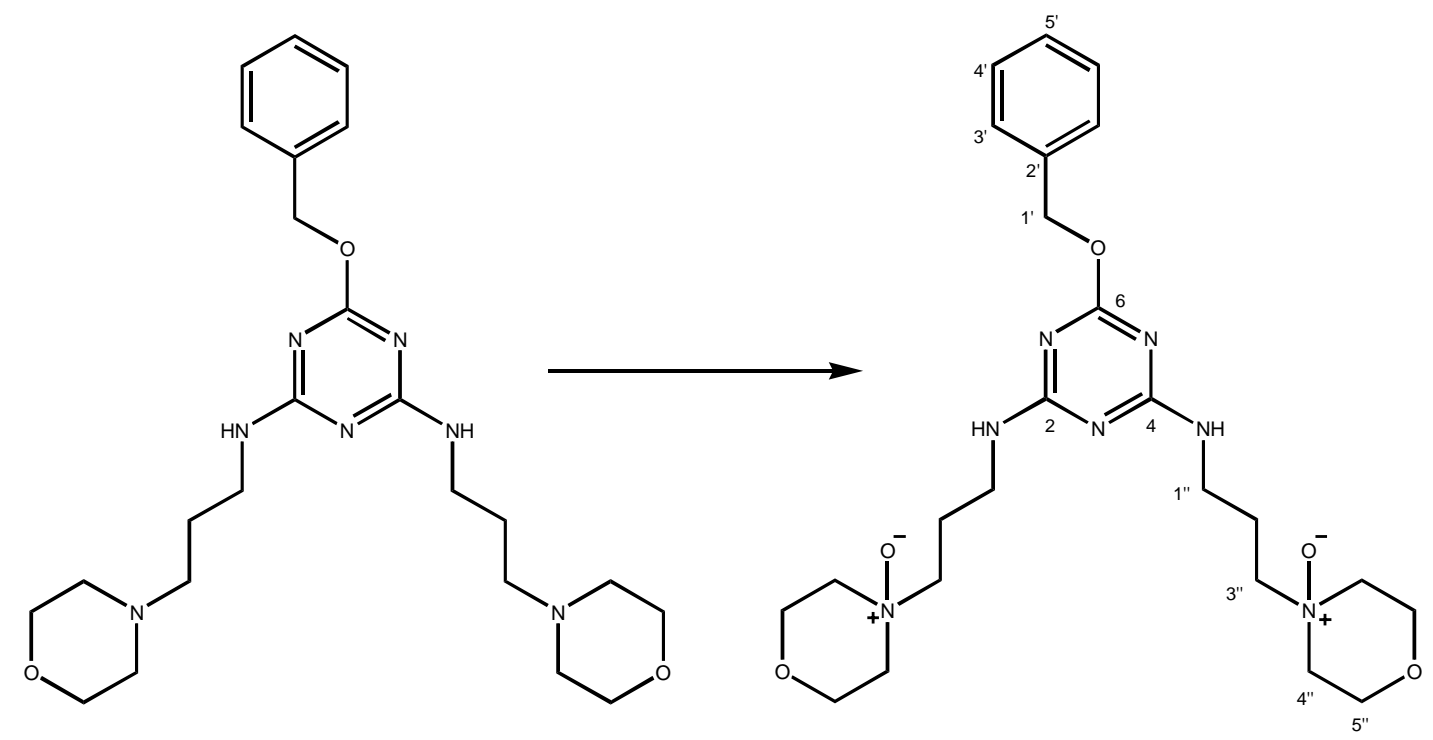

Scheme 2 Reagents and Conditions: $m$-CPBA, DCM, $-78^{\circ} \mathrm{C}(72 \%)$

Preparation of 6-benzyloxy- $N, N^{\prime}$-bis(3-morpholin-4ylpropyl)[1,3,5]triazine-2,4diamine.

To a stirred solution of 2-benzyloxy-4,6-dichloro[1,3,5]triazine (2.56 g, $10.00 \mathrm{mmol})$ in THF $(60 \mathrm{ml})$, was added DIPEA $(2.84 \mathrm{~g}, 22.00 \mathrm{mmol})$ followed by the dropwise addition of 4-(3-aminopropyl)morpholine (3.18 g, $22.00 \mathrm{mmol})$. After $16 \mathrm{~h}$ at $60{ }^{\circ} \mathrm{C}$ the reaction was cooled to room temperature and the solvent removed under reduced pressure. The reaction components were added to water $(60 \mathrm{ml})$ and extracted into ethyl acetate $(50 \mathrm{ml} \times 2)$, before drying the combined organic phase $\left(\mathrm{MgSO}_{4}\right)$, filtering and removing the solvent under reduced pressure. Analysis by silica TLC (10\% methanol-DCM) showed three spots by UV, the largest of which, $\mathrm{R}_{\mathrm{f}} 0.38$ corresponded to desired product. Purification by silica flash chromatography (10\% methanol-DCM) provided the title product as a colourless oil which solidified to a white solid on standing (2.76 g, 59\%); m.p. $102-104{ }^{\circ} \mathrm{C}$; $v_{\max }$ (thin film): 3255, 2943, $1512,1414,1335,1114,1067,812,729 \mathrm{~cm}^{-1} ; \delta_{\mathrm{H}}\left(\mathrm{CDCl}_{3}, 400 \mathrm{MHz}\right): 7.38(2 \mathrm{H}, \mathrm{br} . \mathrm{s}$, 
CH-4'), 7.36-7.29 (3H, m, CH-3', 5'), 6.50-5.95 (2H, 4 x br. s, rotamers, $\mathrm{NH})$, 5.38$5.25\left(2 \mathrm{H}, 2\right.$ x br. s, rotamers, $\mathrm{CH}_{2}-1$ '), 3.67 (8H, br. s, $\mathrm{CH}_{2}-5$ "), 3.44 (4H, br. s, $\mathrm{CH}_{2-}$ 1"), 2.35 (12H, br. s, $\left.\mathrm{CH}_{2}-3 ", 4 "\right), 1.70$ (4H, br. s, $\left.\mathrm{CH}_{2}-2 "\right) ; \delta_{\mathrm{C}}\left(\mathrm{CDCl}_{3}, 100 \mathrm{MHz}\right)$ : 167.0 (1C, C-6), 166.0 (2C, C-2, 4), 137.0 (1C, C-2'), 128.6-128.1 (5C, CH-3', 4', 5'), 69.0 (1C, $\left.\mathrm{CH}_{2}-1^{\prime}\right), 67.3$ (4C, $\left.\mathrm{CH}_{2}-5 "\right) 57.4$ (2C, $\left.\mathrm{CH}_{2}-3 "\right), 54.0$ (4C, $\left.\mathrm{CH}_{2}-4 "\right), 40.0$ (2C, $\left.\mathrm{CH}_{2}-1 "\right), 28.0$ (2C, $\left.\mathrm{CH}_{2}-2 "\right) ; \mathrm{m} / z$ (+ve LSIMS, 3-NBA): $472\left(\mathrm{MH}^{+}, 100 \%\right)$. Found $\left(\mathrm{MH}^{+}\right)$: 472.3020. $\mathrm{C}_{24} \mathrm{H}_{38} \mathrm{~N}_{7} \mathrm{O}_{3}$ requires 472.3036. Crystals suitable for X-ray analysis were prepared by slow diffusion of petroleum ether $\left(60-80^{\circ} \mathrm{C}\right)$ into a solution of 6benzyloxy- $N, N^{\prime}$-bis(3-morpholin-4-ylpropyl)[1,3,5]triazine-2,4-diamine in chloroform (data to be reported elsewhere). 
Preparation of 6-benzyloxy- $N, N^{\prime}$-bis(3-morpholin-4-ylpropylamine oxide) $[1,3,5]$ triazine.

To a stirred solution of 6-benzyloxy- $N, N^{\prime}$-bis-(3-morpholin-4yl-propyl)[1,3,5]triazine2,4-diamine $(1.10 \mathrm{~g}, 2.33 \mathrm{mmol})$ in DCM $(40 \mathrm{ml})$ at $-78^{\circ} \mathrm{C}$, was added potassium carbonate $(0.96 \mathrm{~g}, 7.00 \mathrm{mmol})$ followed by the dropwise addition of $50 \% \mathrm{~m}$-CPBA (1.61 g, $4.66 \mathrm{mmol})$ in DCM $(5 \mathrm{ml})$. After $2 \mathrm{~h}$ at $-78{ }^{\circ} \mathrm{C}$ the reaction was allowed to warm to room temperature. 2-Methylpropene was bubbled through the reaction mixture $(5 \mathrm{~min})$ before removal of the potassium carbonate by vacuum filtration. After washing the potassium carbonate with THF (40 ml) the solvent was removed from the filtrate under reduced pressure. Analysis by alumina TLC (10\% methanolDCM) showed three spots $R_{f} 0.64$ (starting material), $R_{f} 0.25$ (desired product) and $R_{f}$ 0.00 ( $m$-CPBA) by UV. Purification by gradient neutral alumina chromatography ( 0 $2 \%$ methanol-DCM) provided the title product as a white foam $(0.85 \mathrm{~g}, 72 \%) ; v_{\max }$ (solid): $3245,2939,1545,1441,1339,1112,918,857,811,722 \mathrm{~cm}^{-1} ; \delta_{\mathrm{H}}\left(\mathrm{CDCl}_{3}, 400\right.$ MHz): 7.40 (2H, br. s, CH-4'), 7.38-7.28 (3H, m, CH-3', 5'), 7.22-6.90 (2H, 2 x br. s, rotamers, $\mathrm{NH}), 5.39-5.20\left(2 \mathrm{H}, 2 \mathrm{x}\right.$ br. s, rotamers, $\left.\mathrm{CH}_{2}-1^{\prime}\right), 4.39\left(4 \mathrm{H}, \mathrm{t} J 11.0, \mathrm{CH}_{2}-\right.$ 5" $\left.{ }_{\mathrm{ax}}\right), 3.75\left(4 \mathrm{H}, \mathrm{d} J 12.0, \mathrm{CH}_{2}-5_{\text {eq }}\right.$ ), 3.51 (4H, br. s, $\left.\mathrm{CH}_{2}-1 "\right), 3.29$ (4H, br. s, $\left.\mathrm{CH}_{2}-3 "\right)$, $3.18\left(4 \mathrm{H}\right.$, br. s, $\mathrm{CH}_{2}-4{ }^{\prime \prime}$ ) $, 3.10\left(4 \mathrm{H}, \mathrm{d} J 10.5, \mathrm{CH}_{2}-4{ }^{\prime \prime}\right.$ ) $), 2.15$ (4H, br. s, $\left.\mathrm{CH}_{2}-2 "\right) ; \delta_{\mathrm{C}}$ $\left(\mathrm{CDCl}_{3}, 100 \mathrm{MHz}\right): 167.0$ (1C, C-6), 166.0 (2C, C-2, 4), 137.0 (1C, C-2'), 128.7128.3 (5C, CH-3', 4', 5'), 69.9 (2C, $\left.\mathrm{CH}_{2}-3 "\right), 68.3$ (1C, $\left.\mathrm{CH}_{2}-1^{\prime}\right), 64.6$ (4C, $\left.\mathrm{CH}_{2}-4 "\right)$, 62.0 (4C, $\left.\mathrm{CH}_{2}-5 "\right), 38.7$ (2C, $\left.\mathrm{CH}_{2}-1 "\right), 22.6$ (2C, $\left.\mathrm{CH}_{2}-2 "\right) ; \mathrm{m} / z$ (+ve LSIMS, glycerol): $504\left(\mathrm{MH}^{+}, 40 \%\right)$. Found $\left(\mathrm{MH}^{+}\right):$504.2940. $\mathrm{C}_{24} \mathrm{H}_{38} \mathrm{~N}_{7} \mathrm{O}_{5}$ requires 504.2934. 